\title{
Strategic Approaches to obtain Financial Benefits when using Information Technology
}

\author{
Elena MIRCEA \\ The Bucharest University of Economic Studies, Bucharest, Romania \\ elenamircea94@gmail.com
}

Received date: 3 September 2020; Accepted date:21 December 2020; Published date: 2 February 2021

Academic Editor: Maja Pušnik

Copyright (C 2021. Elena MIRCEA. Distributed under Creative Commons Attribution 4.0 International CC-BY 4.0

\begin{abstract}
This last year completely changed humanity and the life we were used to, coming with economic and social challenges. The COVID-19 outbreak reminded humanity that people are not immune to any type of catastrophe, emphasizing the importance of adaptability. Having an agile business is mandatory in order to remain competitive on the market and successful changes are maintained by a strong infrastructure that ensures the operativity. Major investments are needed in productive work and efficient processes, analyzing the overall performance of the company. The aim of this paper is to present the process and the benefits of managing changes in the financial sector when a company is using highperformance information systems, by using specific examples. Information Technology can save a business, however implementing and accepting specific principles and practices can be a hard challenge for the involved people. Digital transformation can bring benefits when IT standard processes are compliant, but can also fail because of not spending enough time analyzing and changing people's behaviors and organizational culture. Agility consists in the ability to adapt in real time to the new challenges and to embrace changes without impacting the business processes.
\end{abstract}

Keywords: Information Systems, Change Management, Business Strategies, Agile.

\section{Introduction}

The predecessor of information systems is considered to be Hollerith's tabulating machine. Since 1890 when Herman Hollerith developed the tabulating machine, offering a major step to automatization, information systems have known different stages (Zwass, 2011). An information system is an integrating set of hardware and software components designed for collecting, storing, analysing and processing data. Around 1960 was the time when a new domain appeared on the market, "Management Information System" (MIS) describing how information technology, management, finance and accounting can mix together, obtaining an

Cite this Article as: Elena MIRCEA (2021)," Strategic Approaches to obtain Financial Benefits when using Information Technology ", Journal of Eastern Europe Research in Business and Economics Vol. 2021 (2021), Article ID 129455, DOI: 10.5171/2021.129455 
innovative system that can define the success of a company. Only in the late '70s worldwide companies were able to utilize the power of those systems, when some IS software versions for personal computers were released (Hirschheim \& Klein, 2012).

In over 60 years of existence, information systems had a spectacular evolution, creating a new domain, different from both information technology and business, but combining knowledge from several fields. The main components come from IT (computer hardware, software and telecommunications and databases) and from the financial sector (principles, practices and procedures). Business relies on this integrated set of components for collecting, storing and processing data in order to carry out specific operations, customer support and remain competitive on the market. IT development has been occurring under Moore's law, the power of the computer devices has been doubling every 18-24 months (Gregersen, 2011), so in order to stay on the market every company needs to measure the organizational performance and the impact of any change done in the environment.

The literature (Zwass, 2011) presents three types of information systems, grouped in a pyramidal structure: operational systems - the base of the structure, support of knowledge work - the middle layer and the management support - top layer. Transactional Processing Systems support the processes through which products are designed, produced and sold, using large integrated systems as ERP, CRM and SCM. Knowledge work stands for manipulating abstract content and theories through professional support systems, collaboration systems and knowledge management systems. Top managers use specific systems as decision support systems or executive information systems, systems that rely on TPS for operations.

\section{Managing Changes for Increasing Organizational Performance}

It is widely accepted that the appearance of information systems came as a response to the need of automatization and standardization of all the already existing business processes and practices. Financial success depends on how thoroughly people inside the organization embrace the change, so creating a new organization design, new processes and implementing new technologies may never be seen as an improvement if managers do not bring individuals along.

IT Service Management is the concept that describes the principles and practices used by an organization to maximize business value, using the information technology. Accomplishing international, national and industrial standards, ITSM describes IT as the key concept in developing a reliable business. Besides being a standard, ITSM became a culture, a mindset that guides managers in achieving the desired outcome. Forbes Insights published in collaboration with BCM an analysis done over the state of ITSM presenting also the most used frameworks and processes (Forbes Insights \& BMC, 2017): 


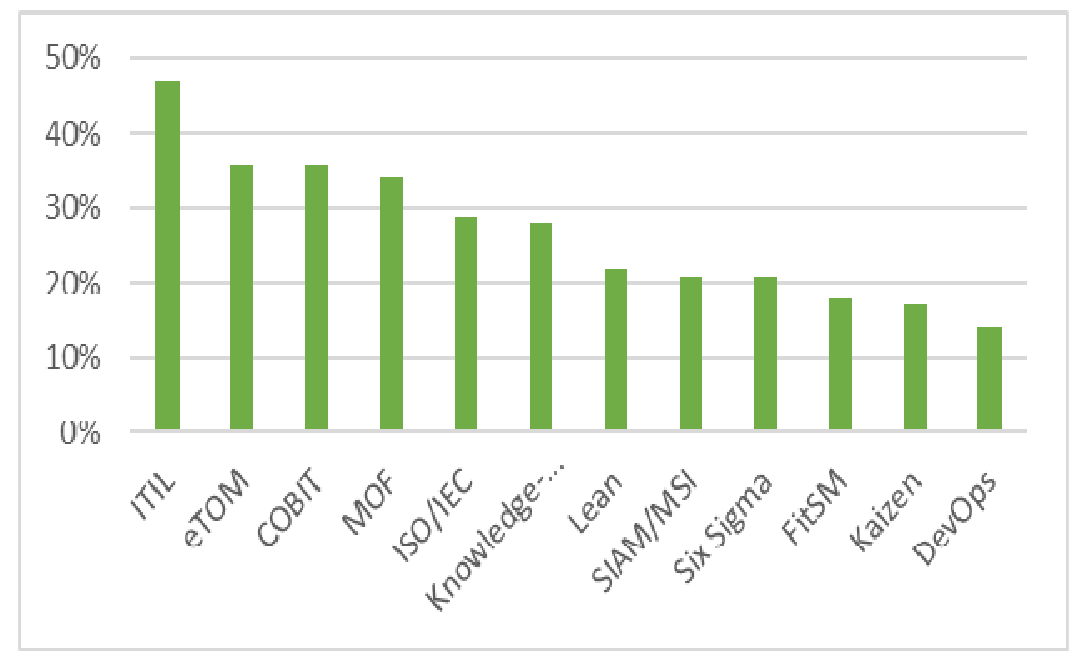

Figure 1: ITSM Frameworks Popularity (adaptation after (Forbes Insights \& BMC, 2017))

The top key performance indicator to measure ITSM effectiveness is Information Technology Infrastructure Library, with over $47 \%$ of the market, it is a best practice framework that "gives guidance on the provision of quality IT services and the processes, functions and other capabilities needed to support them" (AXELOS, 2011). ITIL processes are organized around five service lifecycle stages (Lindros, 2017):

- Service Strategy - mapping the IT model over the organizational structure, establishing processes for monitoring,

- Service Design - continuous improvement for IT processes and services to best fit organization needs,

- Service Transition - defines and mitigates the risk through change management process,

- Service Operation - daily support for functional operations,

- Continual Service Improvement KPIs evolution, problems and optimization proposals.

In the 90's, COBIT was designed as a set of IT control objectives and later ISACA founded the idea of IT management and information governance. By helping managers to align business goals with IT practices, COBIT is establishing the link between information systems, business and information technology. Through its five components, COBIT framework offers metrics that measure the achievement while identifying new business responsibilities and ways of accomplishing them using IT processes and minimizing risk (Simplilearn, 2020). The process description acts as a reference model, but also as a common language and by controlling the objectives, the auditors have a clear list of requirements that have to be checked (Information System Audit and Control Association, 2019).

ITIL comes at the same level with COBIT, without excluding the other one. While COBIT explains what should be done, ITIL offers solutions for how to do IT governance in a correct way.

Having the same popularity as COBIT, Business Process Framework (eTOM) delivers a widely agreed and adopted service-oriented architecture especially for telecommunications and entertainment industry. The conceptual level of eTOM has three major areas, reflecting the main focuses within enterprises (tmforum, 2020):

- Strategy, Infrastructure and Product covering planning and lifecycle management;

Elena MIRCEA, Journal of Eastern Europe Research in Business and Economics, DOI: $10.5171 / 2021.129455$ 
- Operations presented as the core of day-to-day operational management;

- Enterprise Management describing corporate or business support management.

The framework can be utilized as a common language between companies, departments or teams when discussing process issues, defining process flow to model and standardize interactions and to define requirements for the IT systems that would be supporting the process flows.

Business Process Framework can be linked with ITIL practices, being described as complementary frameworks that support each other on the official forum (tmforum, 2020).

Change management includes the processes, tools and techniques used to achieve desired outcomes from a business perspective, but focusing also on helping people adopt and utilize the change in dayto-day work. The notion defines a process, but also a competency and everything is driven by a change manager who ensures that every change is documented, tested and understood by the application users.

This paper focuses on understanding the impact of changes in daily business by following a repeatable cycle and using a set of tools that increase organizational effectiveness and measure the performance of the companies. Hiatt and Creasey established in (Hiatt \& Creasey, 2003) the 5 tenets of change:

- We change for a reason;

- Organizational change requires individual change;

- Organizational outcomes are the collective result of individual change;

- Change management is an enabling framework for managing the people side of change;

- We apply change management to realize the benefits and desired outcomes of change.
On Prosci's blog, each tenet is argued with real life examples (Creasey, 2019). For only $26 \%$ of the organizations, the reason for the change is clear, but the biggest concern is raised by the fact that individuals don't understand that their mindset and the way they currently do their work have to change completely. $91 \%$ of the webinar participants organized by Prosci answered that those crucial individual changes were not defined in their organization (Creasey, 2019). Planning a change on Monday and the go-live on Tuesday is not change management, people need time to understand and adapt their work.

Managing a business can be really challenging; using a wrong configured information system and bringing the agility required by this pandemic situation, without understanding it can turn a successful company into a failure overnight. Business models have to be taken into account and according to the Japanese learning method, SHU-HA-RI (IBM Agile Explorer, 2018), you have to know the stage of your business before implementing any major change. SHU (follow) is the first phase; in which a new business arises and is recommended to understand and respect the previous business examples, following the good practices and learning from other companies' mistakes. HA (break) is the intermediate stage in which some more adjustments can be done to the standard processes. RI (transcend) final stage, with a mature and experienced management, adaptability to changes based on trust and communication.

When it comes to technology, the future is marked by Cloud Computing and cloud applications. Many companies are already there and invested in this new technology that brings IT closer to the users. Cloud Computing defines on-demand availability of computer system resources without direct user involvement. Based on the service models' classification (SaaS, PaaS and IaaS), evolved the paradigm "Everything as a Service" (XaaS) presenting the new market trends, when the customer pays only for the ordered services; a

Elena MIRCEA, Journal of Eastern Europe Research in Business and Economics, DOI: $10.5171 / 2021.129455$ 
service for which no maintenance and personal involvement is needed.

In 2012, "Journal of Information Systems" published an article, mentioning "Information System as a Service" (Hilman, 2012), an era in which companies use a product and manage only its interface, but all the infrastructure, databases and firewall rules are maintained by different providers. Today, the future of the outsourcing companies could be under pressure taking into consideration the pandemic situation, if the main companies are not getting enough business, they would not be able to pay for the outsourcing services. The IT outsourcing companies have adapted quickly, offering constant support even from home offices, but all their income is based on the primary companies and their ability to sustain business during tough times.

An agile company is characterized by the ability to recognize disruptive factors and to respond quickly in a changing and turbulent environment. Agility measurement is the time between the appearance of an opportunity and its capitalization, the shorter the time, the more agile is the company. Big companies such as Oracle, SAP, Microsoft relied on Cloud Computing, offering all their services as packages that can be accessed over the internet. The customer has all the advantages of an integrated system, without knowing the infrastructure behind it.

Patrick Lencioni described in (Lencioni, 2002) the five dysfunctions of a team, by disposing them in a pyramidal form. By analyzing the dynamic inside of a group and gathering the common mistakes, he concluded that at the base of the pyramid can be found the lack of trust, followed by fear of conflict, lack of commitment, avoidance of responsibilities and in the end the team has no result. All those human reactions and feelings make the acceptance of the change even more difficult and at this level constant work and involvement from the management part is needed.

\section{Strategic Implementation of IS in Business}

According to Forbes, $84 \%$ of companies fail at digital transformation because they do not spend enough time analyzing and changing people's behaviors, culture and the way in which the decisions are taken: "One of the most basic impediment to moving forward on the road to digital transformation is whether or not enough people within the organization are aware of the challenges." (Rogers, 2016).

A good business example when analyzing digital transformation is "Starbucks"; a company that managed to overcome the financial crisis from 2008 by implementing a powerful information system (Thomas, 2014). The worldwide coffee chain suffered important financial losses during the crisis and in order to stay on the market and remain competitive, they had to rethink the whole business strategy together with all their processes. As part of the new strategies, some products had to be retired from the market, and for others, price reductions were inevitable. But the biggest question is what to retire, how do you know what exceeds the average budget of a regular consumer and what is affordable? In order to make the best decision in a short period of time, large volumes of data, gathered from all the stores had to be analyzed. This was the time when the management team decided they needed help, extracting relevant data from all the information they had was impossible without a transactional processing system. Today's success confirms the decision from the past, in 2008 the company was present in 49 countries, owning 17000 cafes (Starbucks Corporation, 2008) and later reaching 31256 locations in 2019, being on the market in 78 countries (Starbucks Corporation, 2019). The case represents an example of an agile management prepared to assume important decisions even when financial indicators would say that it is not the proper time for a costly investment. A good change management determined the rapid adaptation to the new customer requirements. 
Over the last 20 years many companies decided to implement an information system as the benefits are hard to ignore. It was observed that an IS can help companies to save money, increase competitiveness and improve performance. After implementing an IS, FedEx moved 3.4 million packages daily inside the United States and UPS over 15 million all over the world (Thomas, 2014). Increasing organizational performance through information technology is a common practice and many companies are choosing to implement an Enterprise Resource Planning system, which contains integrated modules for finance and accounting, human resources, production planning, sales and distributions, material management and marketing. This integrated program runs one database that is accessible for all business units inside a company and is commonly structured to three tier application architecture: database layer, application server and client presentation layer.

An ERP goal is serving the core business processes, by combining as many functions as possible, enabling communication between different departments and simplifying the process flow. However, the success rate of the implementation is very low and could become worse when an organization manages to obtain the IS benefits and the effects do not last for a long period of time (Ming-Tien Tsai, et al., 2011). Changes and variations of the environment can affect the efficiency of the systems if the ERP is not able to adapt to the modifications. Robbins-Gioia published in 2002 a survey result, made on a hundred enterprises, discovering that $46 \%$ of those companies were unable to maintain the competitive advantages provided by an ERP for a long period of time (Ming-Tien Tsai, et al., 2011). Also, the Conference Board conducted a survey revealing that $40 \%$ of ERP projects fail in one year after go-live (Ming-Tien Tsai, et al., 2011).

Information systems' agility consists in the ability to adapt in real time to the new challenges that can appear in the business environment and to embrace changes without any major impact for organizations. A double approach of agile information system benefits is presented in table 1 .

\section{Table 1: IS Benefits - Double Approach}

\begin{tabular}{|c|c|}
\hline Domain & Benefit \\
\hline \multirow{9}{*}{ Business } & Standard processes and practices \\
\hline & Increase in efficiency of data processing \\
\hline & IS capacities aligned with the business needs \\
\hline & Innovation in technical solutions \\
\hline & Minimizing the risk when implementing changes \\
\hline & Business processes can change in real time \\
\hline & Financial benefits, increase in sales value \\
\hline & Maintenance cost reduction \\
\hline & Faster development of products/services \\
\hline \multirow{7}{*}{$\begin{array}{l}\text { Information } \\
\text { Technology }\end{array}$} & Minimizing the time when implementing changes \\
\hline & Faster recovery in case of outages and downtimes \\
\hline & Data validity improvement \\
\hline & Rapid development of new functionalities \\
\hline & Standardization to internationl standards \\
\hline & Adaptation for system upgrades \\
\hline & Rapid response to security threats \\
\hline
\end{tabular}

Elena MIRCEA, Journal of Eastern Europe Research in Business and Economics, DOI: $10.5171 / 2021.129455$ 
Some difficulties can be mentioned when talking about agility and changes; on the top of the list sits ppeople's reluctance to change, lack of trust in new methods and in others, as human interaction is the key, good communication and strong collaboration is needed, also iinvolvement of all company's departments in the scheduled changes that affect the system is essential to ensure success of that change. A series of assumptions have to be defined from phase one of the change implementation: openness, communication and collaboration, acceptance of the changes and of the new technologies, willingness to help the others in defining and understating a certain process.
George Polya defines in (Polya, 1945) the four principles of solving problems (table 2 ), later adopted in all the fields and more important in business when it comes to link it with information technology. Those four concepts could be adapted when implementing and managing an organizational change. Similarities can be found between Polya's heuristic and agile principles, work and learn based on repetition: listen, iterate, learn and correct; leading to successful implementations while people understand the necessity of the change and manage to learn how to work with the new functionalities.

Table 2: Polya's Heuristic for Solving Problems

\begin{tabular}{|l|l|}
\hline Principle & Approach \\
\hline Understand the problem & $\begin{array}{l}\text { The managers cannot solve the problem simply because they don't } \\
\text { understand it. Without having all the information, it is not possible } \\
\text { to find a proper solution. }\end{array}$ \\
\hline Devise a plan & $\begin{array}{l}\text { Choose the appropriate strategy and this may include using a } \\
\text { predefined model, drawing schemas, looking for a pattern. }\end{array}$ \\
\hline Carry out the plan & $\begin{array}{l}\text { Persist with the chosen plan and only after not seeing the desired } \\
\text { results you can choose another model }\end{array}$ \\
\hline Look back & $\begin{array}{l}\text { Learn from the past mistakes, reflect on what worked ok and what } \\
\text { could be improved. }\end{array}$ \\
\hline
\end{tabular}

Even the agile rituals, the four well-known regular meetings, are based on the same heuristic:

- Sprint Planning - understanding the existing problems and bottlenecks,

- Daily Stand-up - planning and deciding the daily strategy in order to continue the work,

- Sprint Review - following the chosen solution and presenting the sprint result,

- Retrospective - lessons learned, reflect over the last sprint and decide what improvements can be done.

\section{Conclusions}

In conclusion, with a relative short history, information systems managed to influence both finance and information technology markets, by creating a link between business and IT. Managers must understand the necessity of focusing on data flow and collaboration between different departments, mapping processes and practices and less on creating a powerful system only from a technical perspective.

The Internet era opened a new world full of opportunities. A good change management helps organizations to become even more competitive in an unstable environment, by

Elena MIRCEA, Journal of Eastern Europe Research in Business and Economics, DOI: $10.5171 / 2021.129455$ 
adapting their business strategies to the market requirements. Every company should follow the trend when it comes to technology solutions that improve the business overall, but the involved changes have to be thoroughly analyzed. Cloud Computing is the technology on which the biggest IT service providers relied during the past few years and it seems to be the future of an appropriate system management.

Technology can help solving the existing problems, can bring financial benefits and can improve organizational performance, but is a common effort that has to be done by all the involved parties in adapting and evolving to the new processes and practices imposed by an information system.

In the end of the study some limitations have to be mentioned, as the paper focuses more on change management general principle and practices and less on offering a well-defined methodology and "how to" steps. Every company has to find what best fits depending on their needs and must consider the "best practices" as a starting point. The IS implementation or changing effects have to be analyzed by every organization and decide which factors might affect the overall performance.

\section{References}

- AXELOS, 2011. ITIL glossary and abbreviations, s.l.: s.n.

- Creasey, T. J., 2019. Webinar Recap: The 5 tenets of change. [Online] Available at:

https://blog.prosci.com/webinarrecap-five-tenets-of-change

- $\quad$ Forbes Insights \& BMC, 2017. The State of IT Service Management. Delivering Value to Today's Digital Enterprise.

- Gregersen, E., 2011. Encyclopaedia Britannica. [Online] Available at: https://www.britannica.com/technolo gy/Moores-law

[Accessed May 2020].

- Hiatt, J. M. \& Creasey, T. J., 2003. Change Management: The People Side of Change. s.l.:Prosci.
- Hilman, M., 2012. INFORMATION SYSTEM AS A SERVICE: ISSUES AND CHALLENGES. Journal of Information Systems, 8(2), pp. 71-77.

- Hirschheim, R. \& Klein, H. K., 2012. A Glorious and Not-So-Short History of the Information Systems Field. Journal of the Association for Information Systems, 13(4), pp. 188-235.

- IBM Agile Explorer, 2018. Think Academy. [Online] Available at: https://agileibm.mybluemix.net/courses/taste-ofagile/agile-principles-values-andbehaviors

[Accessed January 2019].

- Information System Audit and Control Association, 2019. Effective IT Governance at Your Fingertips. [Online]

Available at: https://www.isaca.org/resources/cobi $\underline{\mathrm{t}}$

[Accessed April 2020].

- Lencioni, P., 2002. The Five Dysfunctions of a Team. s.l.:John Wiley \& Sons, Inc..

- Lindros, K., 2017. CIO from IDG. [Online]

Available at:

https://www.cio.com/article/243893

1 /governanceit-governance-definitionand-solutions.html

- Ming-Tien Tsai, Eldon Y. Li, Kou-Wei Lee \& Wen-Hui Tung, 2011. Beyond ERP Implementation: The Moderating Effect of Knowledge Management on Business Performance. Total Quality Management and Business Excellence, 22(2), pp. 131-144.

- Polya, G., 1945. How To Solve It. s.l.:Princeton University Press.

- Rogers, B., 2016. Why 84\% Of Companies Fail At Digital Transformation. Forbes.

- Simplilearn, 2020. Simplilearn. [Online] Available at: https://www.simplilearn.com/whatis-cobit-significance-and-frameworkrar309-article

[Accessed April 2020].

- Starbucks Corporation, 2008. Starbucks Annual Report. [Online]

Elena MIRCEA, Journal of Eastern Europe Research in Business and Economics, DOI: $10.5171 / 2021.129455$ 
Available at:

http://www.annualreports.com/Hoste dData/AnnualReportArchive/s/NASD AQ SBUX 2008.pdf

[Accessed March 2020].

- Starbucks Corporation, 2019. Starbucks FY19 Annual Report. [Online] Available at: https://investor.starbucks.com/financi al-data/annual-reports/default.aspx [Accessed March 2020].

- Thomas, M., 2014. The benefits of Using Information Systems in Business. Ashford University.

- tmforum, 2020. tmforum.org. [Online] Available https://www.tmforum.org/business- process-framework/

[Accessed September 2020].

- tmforum, 2020. tmforum.org. [Online] Available at: https://www.tmforum.org/businessprocess-framework/relationship-toitil/ [Accessed April 2020].

- Zwass, V., 2011. Encyclopaedia Britannica. [Online] Available at: https://www.britannica.com/topic/inf ormation-system

[Accessed June 2020]. 